\title{
Electromagnetic Phenomena and Collider Performance at Ultra-Relativistic Energies
}

\author{
M.J. Rhoades-Brown
rator Development Department \\ M.J. Rhoades-Brown
Accelerator Development Department \\ Brookhaven National Laboratory \\ Upton, NY 11973, USA
}

CONF-890387--5

DE89 010174

C. Bottcher and M.R. Strayer

Physics Division, Oak Ridge National Laboratory

Oak Ridge, TN 37831, USA

Work performed under the auspices of the U.S. Department of Energy.

\section{DISCLAIMER}

This report was prepared as an account of work sponsored by an agency of the United States Government. Neither the United States Government nor any agency thereof, nor any of their employees, makes any warranty, express or implied, or assumes any legal liability or responsibility for the accuracy, completeness, or usefulness of any information, apparatus, product, or process disclosed, or represents that its use would not infringe privately owned rights. Reference herein to any specific commercial product, process, or service by trade name, trademark, manufacturer, or otherwise does not necessarily constitute or imply its endorsement, recommendation, or favoring by the United States Government or any agency thereof. The views and opinions of authors expressed herein do not necessarily state or reflect those of the United States Government or any agency thereof. 


\section{Introduction}

The Relativistic Heavy Ion Collider (RHIC) at Brookhaven [1] will accelerate fully stripped heavy ions up to ${ }^{197} \mathrm{Au}$ at an energy/nucleon of $100 \mathrm{GeV}$. At this top energy, the two beams of heavy ions will collide in up to six available experimental halls situated around the ring. Of particular interest to the high energy nuclear physics community, is the possibility of both forming and detecting a new and exotic form of matter known as a quark-gluon plasma. It iz understood that this form of matter has the highest probability of being formed in a central collision.

In addition to this so-called nuclear collision, which of course depletes particles from the beam, it is also important to understand the various components of the beam-beam interaction during beam crossing, due to the strong, long range electromagnetic forces between heavy ions [2]. Of course, these effects are peripheral in nature.

At increasing relativistic energies, the electromagnetic field associated with the fully stripped heavy-ions becomes sharply peaked in a direction transverse to the heavy-ion motion. In the laboratory frame, at impact parameters of a few nuclear radii, transverse electric fields in excess of a few $\mathrm{GeV} / \mathrm{fm}$ [2] may be expected for heavy-ions at the highest energies available to RHIC. Under these conditions, we may expect copious production of electron-positron pairs, $[3,4]$ as well as internal electromagnetic excitation of the nuclear dipole band resonances [5].

The new physics associated with strong and pulsed electromagnetic fields from heavy ions has important practical implications for detector design and machine performance at RHIC. For this reason, it is important to develop reliable theoretical models of Quantum Electromagnetic phenomena at ultra-relativistic energies, and to make detailed experimental studies for comparison with these calculations.

In this paper we discuss to the two dominant electromagnetic processes that 
can lead to beam depletion in RHIC. The single largest nuclear depletion mechanism of the RHIC beam is estimated to be the electron capture process [1] following pair production during beam crossing. A previous study [6] has obtained a value of $80 \mathrm{~b}$ per beam for $\mathrm{Au}+\mathrm{Au}$ using the Weizsācker-Williams method [7]. However, a range of values has been advocated by other authors [8]. A recent study has resolved this uncertainty [9]. A more difficult problem to study is the Coulomb disassociation of the heavy ions, following electromagnetic excitation of internal multiple nuclear dipole resonances during beam crossing. These resonances typically decay via particle emission from the nucleus. This loss mechanism is of primary importance to the beam loss of medium mass nuclei (i.e. ${ }^{63} \mathrm{Cu}$ ) and of secondary importance to the beam loss of heavy nuclei [1]. We note there are experimental programs at both CERN [10] and the Brookhaven AGS [11] to study this phenomena.

In section II we present our recent work on electron capture at relativistic collider energies. In section III we discuss the problem of excitation of a single dipole resonance in the perturbative limit, and derive a "rule of thumb" to help judge the validity of this well known perturbative approach for future applications of excitation of multiple resonances. In section IV we discuss the implications of our results for both future atomic and nuclear programs, and the machine performance at RHIC.

\section{Electron Capture at RHIC Energies}

We focus here on the most important aspect of RHIC performance at relativistic energies by presenting calculations for the cross section for electron capture in the two-photon limit, using Monte Carlo integration over intermediate and final momenta [9]. Our calculation is an extension of earlier published work on pair production via two-photon diagrams [3]. The present study spans an energy range 
that includes the present AGS experiments and the future RHIC program. In particular, we compare our results with the equivalent photon or Weizsācker-Williams methods $[7,8]$. We also discuss a more rigorous version of this method, which we call the single peak approximation. This relatively simple expression approximates the full Monte Carlo calculations over a wide energy range.

The electron capture mechanism is shown schematically in the two-photon diagrams of Fig. 1. We use time-dependent perturbation theory to expand the $\mathrm{S}$-matrix in orders of the electromagnetic interactions of the two colliding ions $a, b$. The leading contributions to pair production arise from the two Feynman diagrams, in which each ion interacts exactly once [3]. Capture is described in a sudden approximation, by convoluting the electron line in Fig. 1 into the momentum wavefunction of the final bound state.

Working in natural units $(\hbar=c=m=e=1)$, and assuming a geometry in the CM or collider frame that has the target $(b)$ moving from right to left, parallel to the $\mathrm{z}$-axis with velocity $\beta$; its energy in mass units is $\gamma=\left(1-\beta^{2}\right)^{-\frac{1}{2}}$. The projectile (a) moves from left to right with velocity $-\beta$. All results are presented in terms of the collider energy $\gamma$; the equivalent fixed target energy is given by $\gamma_{F T}=2 \gamma^{2}-1$. The pair production cross section is given by [3].

$$
\sigma_{p a i r}=\frac{1}{(2 \beta)^{2}} \sum_{\sigma_{k}, \sigma_{q}} \int \frac{d^{3} k d^{3} q d^{2} p_{\perp}}{(2 \pi)^{8}}\left|B\left(k, q ; \vec{p}_{\perp}\right)\right|^{2}
$$

where

$$
B\left(k, q ; \vec{p}_{\perp}\right)=A^{(+)}\left(k, q ; \vec{p}_{\perp}\right)+A^{(-)}\left(k, q ; \vec{k}_{\perp}+\vec{q}_{\perp}-\vec{p}_{\perp}\right)
$$

The superscripts $( \pm)$ refer to the direct and crossed diagrams respectively. The notation of (1) is as follows: $\vec{q}, \vec{k}$ are the momenta of the positron and electron in 
the final state, $\vec{p}$ is the intermediate fermion momentum, $\sigma_{q}, \sigma_{k}$ run over the spins of the positron and electron. The label $k$ is short for the quantum numbers $\vec{k}, \sigma_{k}, s_{k}$, where $s_{k}= \pm 1$ for positive and negative energy states. The frequencies of the virtual photons determine the components $p_{0}$ and $p_{z}$ so that only an integration over $\vec{p}_{\perp}$ remains in (1).

The amplitude $A^{(+)}\left(k, q ; \vec{p}_{\perp}\right)$ is expressed as a product,

$$
A^{(+)}\left(k, q ; \vec{p}_{\perp}\right)=F\left(\vec{k}_{\perp}-\vec{p}_{\perp}: \omega_{a}\right) F\left(\vec{q}_{\perp}-\vec{p}_{\perp}: \omega_{b}\right) \tau_{k q}\left(\vec{p}_{\perp}: \beta\right),
$$

where $F$ is the scalar part of the field associated with each heavy-ion,

$$
F(\vec{u}: \omega)=\frac{4 \pi Z}{\vec{u}^{2}+(\omega / \beta \gamma)^{2}},
$$

and $\omega_{a}, \omega_{b}$ are the frequencies associated with the fields of $a, b$ respectively. The amplitude $\tau_{k q}$ relates the intermediate photon lines to the outgoing fermion lines. An expression for $A^{(-)}\left(\vec{k}, \vec{q}, \vec{p}_{\perp}\right)$ is obtained by reversing the sign of $\beta$ and thereby interchanging $\omega_{a}$ and $\omega_{b}$.

The cross section for electron capture is obtained from (1)-(4) by convoluting the amplitude $B$ into the momentum representation of the bound state wavefunction $\Phi_{T}\left(s_{k}, \vec{k}\right)$. Most of the capture is into the $\mathrm{K}-\mathrm{shell}\left(1 s_{1 / 2}\right.$ state), for which $\Phi_{T}$ is independent of the usual Dirac hydrogenic quantum numbers $\sigma_{T}$ and $m_{T}$. In this way, our expression for the capture cross section derived from (1) becomes

$$
\sigma_{c a p}=\frac{1}{(2 \beta)^{2}} \sum_{\sigma_{q}} \int \frac{d^{3} q d^{2} p_{\perp}}{(2 \pi)^{5}}\left|\sum_{\Delta_{k}} \int \frac{d^{3} k}{(2 \pi)^{3}} \tilde{\Phi}_{T}\left(s_{k}, \vec{k}\right) B\left(k, q ; \vec{p}_{\perp}\right)\right|^{2}
$$

where $s_{q}=-1, s_{k}= \pm, \sigma_{q}= \pm$ and $\sigma_{k}=\sigma_{T}$. The bound state wavefunction $\tilde{\Phi}_{T}\left(s_{k}, \vec{k}\right)$ is expressed here in the collider frame, and is reexpressed in terms of $\Phi_{T}$ 
by a Lorentz transformation [9]. We shall evaluate (5) by Monte Carlo methods $[3,9]$.

Equation (5) can be put in a form more suitable for Monte Carlo evaluation by using the fact that $\Phi$ is sharply and symmetrically peaked around $\vec{k}_{z}=0$ or $k_{z}=\beta \gamma\left(1+\vec{k}_{\perp}^{2}\right)^{\frac{1}{2}}$. Under these circumstances (5) can be replaced by

$$
\sigma_{\text {cap }} \simeq \frac{1}{(2 \beta)^{2}} \sum_{\sigma_{k}, e_{1}} C\left(s_{q}\right) \int \frac{d^{3} k d^{3} q d^{2} p_{\perp}}{(2 \pi)^{8}}\left|\tilde{\Phi}_{T}\left(s_{k}, \vec{k}\right)\right|^{2}\left|B\left(k, q ; \vec{p}_{\perp}\right)\right|^{2},
$$

where the renormalization factor is given by

$$
C\left(s_{k}\right)=\left|\int \tilde{\Phi}_{T}\left(s_{k}, \vec{k}\right) d^{3} k\right|^{2} \int\left|\tilde{\Phi}_{T}\left(s_{k}, \vec{k}\right)\right|^{2} d^{3} k
$$

The integral in (6) can be evaluated as it stands, the integrand being positive definite. The error is $0\left(1 / \gamma^{4}\right)$ and hence negligible in the range we consider $(\gamma-3)$.

This single peak approximation is based on the observation that for $\gamma>>1$ the form factor $F$ is sharply peaked around $\lambda_{\perp}=0$, where $\vec{\lambda}_{\perp}=\vec{q}_{\perp}-\vec{k}_{\perp}$ and $\lambda_{z}=q_{z}-k_{z}=\omega_{q} / \beta=\left(E_{q}^{(-)}-E_{T}\right) / \beta$. Then we can separate $[9]$,

$$
\mathcal{F}_{a}=Z_{a}^{2} \int d^{2} \lambda_{\perp}\left(\vec{\lambda}_{\perp} \vec{\lambda}_{\perp}\right)\left|F\left(\vec{\lambda}_{\perp}: \omega_{q}\right)\right|^{2},
$$

and evaluate the rest of the integrand for capture [9] at $\lambda_{\perp}=0$, or

$$
\vec{k}=\vec{q}-\frac{\omega_{q}}{\beta} \vec{e}_{x} .
$$

Within a constant factor, $\mathcal{F}_{a}$ is the equivalent photon flux tensor associated with the projectile [3]. The integral over $\lambda_{\perp}$ in (8) must be cut off at $\lambda_{\perp} \simeq m_{\perp}$, where

$$
m_{\perp}=\left(1+\vec{q}_{\perp}^{2}\right)^{\frac{1}{2}} .
$$


If $(6)$ is evaluated exactly, this cutoff arises naturally. Then we finally obtain

$$
\sigma_{\text {cap }}=\left(\frac{Z_{a} \alpha}{2 \pi \beta}\right)^{2} \sum_{e_{q}} \int \frac{d^{3} q}{\omega_{q}^{2}} p\left(\frac{\beta \gamma m_{\perp}}{\omega_{q}}\right) M(\vec{k}, \vec{q})\left|\Phi_{T}\left(s_{k}, \vec{k}\right)\right|^{2} .
$$

For most purposes $P(x) \sim \ln x ; M$ comes from the spinors, and is of order unity or less.

In Fig. 2, the capture cross section is plotted for $A u+A u$ collision as a function of the collider energy. The scale of the cross section is given by $\sigma_{0}=\left(\lambda Z_{a} Z_{b} \alpha^{2}\right)^{2}$ where $\lambda$ is the Compton wavelength of the electron, and $\alpha$ is the fine structure constant. The value of $\sigma_{0}$ for $A u+A u$ is $164.7 \mathrm{~b}$. Figure 2 shows three calculations: the full Monte Carlo [5], the single peak approximation [9], and a Weizsäcker-Williams calculation from Bauer and Bertulani [8]. The error bars reflect the remaining statistical errors in the Monte Carlo evaluation of the integral. The single peak approximation agrees well with the Monte Carlo over the entire energy range, and is algebraicly simpler. The Weizsäcker-Williams curve is consistently lower than the Monte Carlo method by a factor of three, possibly because of an error in this calculation. An independent calculation by S.Y. Lee and J. Weneser, based on the same assumptions, agrees well with our results [6].

From the perspective of RHIC accelerator performance, the top RHIC energy per nucleon for $\mathrm{Au}$ is $100 \mathrm{GeV}$. At this energy the capture cross section is $72 \mathrm{~b}$ per beam.

In Fig. 3, the capture cross sections are shown for a sample of symmetric heavyion collisions used in the design of RHIC. These are full Monte Carlo calculations. To convey the variation with $Z$, all the results here are scaled according to $\lambda^{2}=$ $1.49 \mathrm{~Kb}$. Very few heavy-ions would be lost to this mechanism for $A \sim 100$. The result for ${ }^{197} \mathrm{Au}$ is in harmony with a ten-hour beam lifetime at RHIC. An equally 
encouraging aspect of Fig. 3 is the saturation at large values of $\mathrm{Z}$ : the electron capture cross section for $\mathbf{U}+\mathrm{U}$ is only twice that for $A u+A u$. It has recently been determined that ${ }^{238} U$ may be injected into RHIC [12].

\section{Coulomb Disassociation at Top RHIC Energies}

This subject has been studied in the literature for a single resonance by several authors [5], but the theory exists only for excitation probability of a single dipole resonance, in the perturbative limit. Applying these formulas to top RHIC energies the dipole cross section $\sigma_{D}$ is 50.0 barns for ${ }^{197} A u+{ }^{197} A u$. Here we critically address the accuracy of this best available nuclear theory, and discuss a "rule of thumb" to help judge the validity of this approach for future applications [2]. Assuming that only forward scattering (scattering angle $=0$ ) is of interest to beam crossing, the probability amplitude $a_{f i}$ of exciting the nuclear dipole resonance is given by $[2,5]$,

$$
a_{f i}=\frac{8 \pi}{\gamma_{F T}} \sqrt{\frac{\pi}{3}} \frac{Z}{R} \times K_{1}\left(\frac{E_{f} R}{\gamma_{F T} \beta \hbar c}\right) \times \sqrt{B E 1}
$$

where $K_{1}(\mathbf{x})$ is a Bessel function, $E_{f}$ is the excitation energy of the single nuclear dipole resonance. $B E 1$ is the strength of the resonance. For values of the nuclear seperation $r<R$, the dipole potential is zero in this simple perturbative approximation. Using the dipole sum rules [5],

$$
\begin{gathered}
E_{f} B E 1=14.8 N Z A^{-1} \\
E_{f}=78 A^{-1 / 3}
\end{gathered}
$$

where $A=N+Z$ and $N$ is the neutron number, the transition amplitude becomes

$$
a_{f i}=\frac{1.16}{\gamma_{F T} R} \sqrt{\frac{\pi^{3} Z^{3} N}{A^{2 / 3}}} \times K_{1}\left(\frac{78 R}{\gamma_{F T} \beta \hbar c A^{1 / 3}}\right)
$$


This. quantity is plotted in Figures $4 \& 5$ for ${ }^{197} A u+{ }^{197} A u$ and ${ }^{63} C u+{ }^{63} C u$ respectively, as a function of $\gamma_{F T}$ or $\gamma_{c o l l}$.

From these figures, it can be seen that $a_{f i}$ becomes a constant at quite small values of $\gamma_{\text {coll }}$. In particular, for $R=20 \mathrm{fm}, 50 \mathrm{fm}$ we find $a_{f i}>1$. Hence for these smaller distances the perturbative formalism for $a_{f i}$ may not be considered accurate. Obviously, as $R$ increases or the nuclear charge decreases the amplitude $a_{f i}$ becomes smaller.

Using a property of Bessel functions $\left(K_{1}(x)=x^{-1} 1 x 1<1\right)$, it is possible to derive a useful "rule of thumb" for the validity of the perturbative approach. In this way the value of the radius for which $a_{f i} \leq 1$ is

$$
R \leq 4 Z^{3 / 4} N^{1 / 4} \quad\left(R \geq 4 Z^{3 / 4} N^{1 / 4}\right)
$$

The value is independent of $\gamma_{\text {coll }}$, as figures 1 and 2 show clearly. For ${ }^{197} A u+{ }^{197}$ $A u$ this value is $R>350 \mathrm{fm}$. For ${ }^{63} \mathrm{Cu}+{ }^{63} \mathrm{Cu}$ we find $R>120 \mathrm{fm}$. Typical perturbative calculations for ${ }^{197} A u+{ }^{107} A u$ start at $R>10 \mathrm{fm}$ [5].

The consequences of these results on RHIC beam-beam interactions are very interesting. The perturbative estimate $\sigma_{D}=50.0$ barns for the first dipole resonance in ${ }^{197} A u$ is expected to be an upper limit. However, for small $R$ values the electromagnetic pulse resembles a delta function, composed of an infinite number of harmonics. This result together with the breakdown of first order perturbation theory for $\sigma_{D}$, implies the possibility exists of exciting several dipole resonances of higher excitation energy. This is a new physical effect and has not been studied in a consistent non-perturbative formalism. However, the width of these new resonances is expected to be quite broad, and so the overall cross section to the first and subsequent dipole resonances (if they exist) is not expected to be much larger 
than the upper limit given by the simple perturbative formalism. 


\section{Discussion}

In this paper we have investigated the strong-field electromagnetic phenomena that is expected to be important in determining the ultimate RHIC performance. Of primary importance is the atomic electron capture process, following pair production from the QED vacuum during beam crossing. We evaluated the leading Feynman diagrams numerically by Monte Carlo methods and attempted to resolve apparent discrepancies among published Weizsācker-Williams estimates for this mechanism. In particular, for point charges, the approximations leading to Weizaäcker-Williams introduce divergences which are removed by cutoffs in the transverse mass. The non-uniqueness of this procedure explains why a wide variety of estimates for the same cross section exist. A capture cross section of 100 barns for this capture process corresponds to a beam loss of 0.032 /hour for ${ }^{197} \mathrm{Au}$ in RHIC. This is not prohibitively large, considering an expected 10 hour beam lifetime.

The Coulomb disassociation mechanism, associated with internal nuclear dynamics, is not well understood at present. Perturbative estimates for a single dipole

resonance of $\sigma_{D}=50$ barns for ${ }^{197} A u+{ }^{197} A u$ correspond to a beam loss rate of only $.0162 /$ hour. It remains to be explored experimentally if multiple dipole excitations are possible, and if the unitarity violating results from perturbation theory, normally quoted for this single dipole mechanism are accurate. Experience with lower energy coupled-channels calculations strongly suggest unitarity violating perturbative estimates are upper limits.

This research was sponsored by the Division of Nuclear Physics of the U.S. Department of Energy under contract No. DE-AC02-76CH00016 with Brookhaven National Laboratory, under contract No. DE-AC05-84OR21400 with Martin Marietta Energy Systems, Inc., and by the Division of Chemical Sciences, Office of 
Basic Energy Sciences. 


\section{$\underline{\text { References }}$}

[1] "Conceptual Design of the Relativistic Heavy-Ion Collider, RHIC" BNL Report No. 51932, May 1986.

[2] M.J. Rhoades-Brown in Proceedings of "European Particle Accelerator Conferencen, Rome, June 1988.

[3] C. Bottcher and M.R. Strayer, Phys. Rev. D39, March 1 (1989).

[4] G. Baur and C. Bertulani, Phys. Rev. C35 (1987) 836.

[5] A. Winther and K. Alder, Nucl Phys. A319 (1979) 518.

[6] S.Y. Lee and J. Weneser, in "Conceptual Design of Relativistic Heavy-Ion Collider, RHIC ${ }^{n}$, BNL Report No. 51932, May 1986, page 134.

[7] C.F. von Weizsācker, Z. Physik 으, (1934) 612.

[8] G. Baur and C. Bertulani, Phys. Rep. 163 (1988) 299.

[9] M.J. Rhoades-Brown, C. Bottcher, M. Strayer, submitted to Phys. Rev. A.

[10] G. Romano, in "Proceedings of The Seventh International Conference on U1-

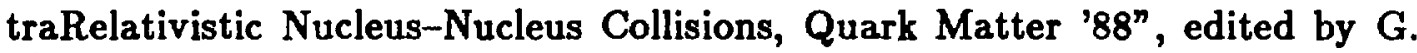
Baym, P. Braun-Munzinger, and S. Nagamiya (North Holland), to be published.

[11] AGS experimental proposal No. 814, M. Fatyga, et al.

[12] M.J. Rhoades-Brown, "Accelerating Uranium in RHIC, I, and II" RHIC Technical Note No. 35 , and 38. 


\section{$\underline{\text { Figure Captions }}$}

Figure 1: Direct (a), and crossed (b), Feynman diagrams for pair production with electron capture into a target state $T$ in a heavy-ion collision.

Figure 2: Scaled capture cross sections for $A u+A u$. The scaling factor $\sigma_{0}=164.7 \mathrm{~b}$. The three curves are: full line, Feynman-Monte Carlo calculations, with statistical uncertainties indicated by error bars; dashed line, the single peak approximation (6); chain dashed line; chain dashed line, Weizsäcker-Williams calculations from [8].

Figure 3: Capture cross sections for symmetric $A_{*}+A_{x}$ collisions, scaled with respect to $\lambda^{2}=1.49 \mathrm{~Kb}$. The curves correspond, as labelled, to the ions $A(Z)=$ $\mathrm{Si}(14), \mathrm{Cu}(29), \mathrm{I}(53), \mathrm{Au}(79), \mathrm{U}(92)$.

Figure 4: Graph of the probability amplitude $a_{f i}$ for exciting the first dipole resonance in ${ }^{197} A u$, as a function of $\gamma_{F T}\left(\equiv \gamma_{c o l l}\right)$. The magnitude of $a_{f i}$ is shown for a range of $R$ values.

Figure 5: As figure 4, but for ${ }^{63} \mathrm{Cu}$. 

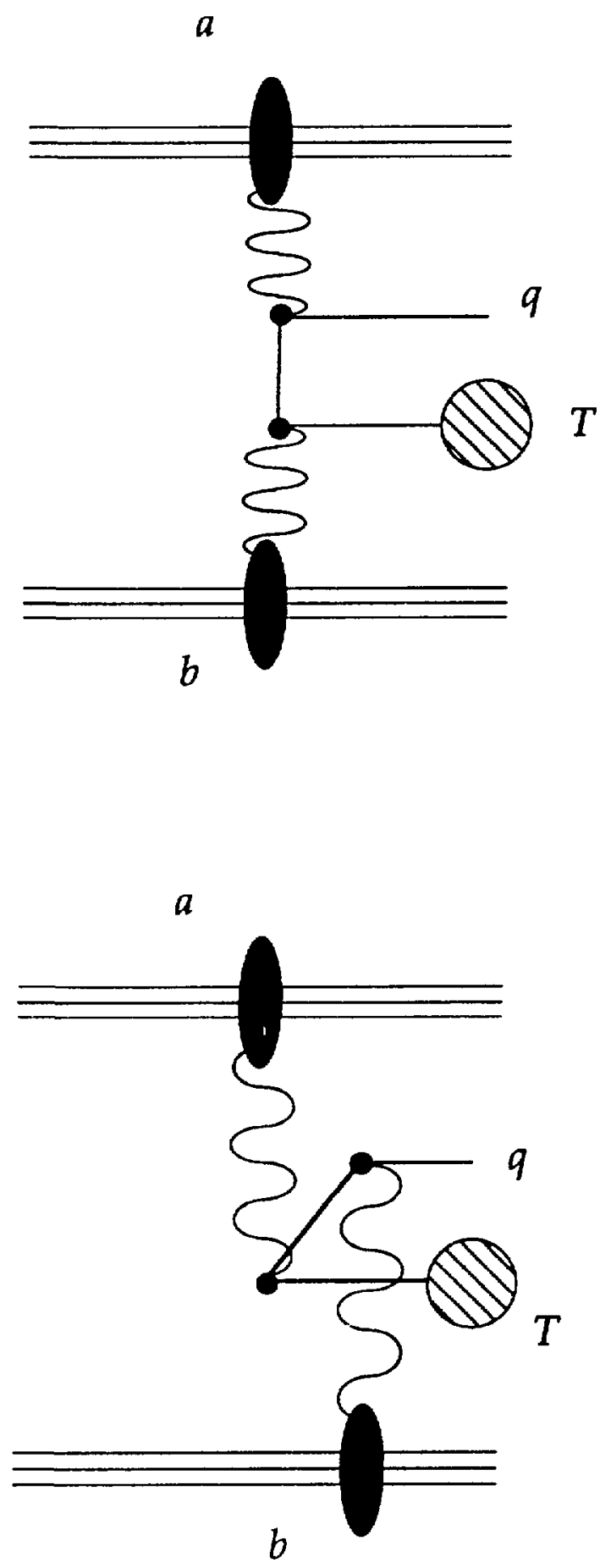

FIG. I 


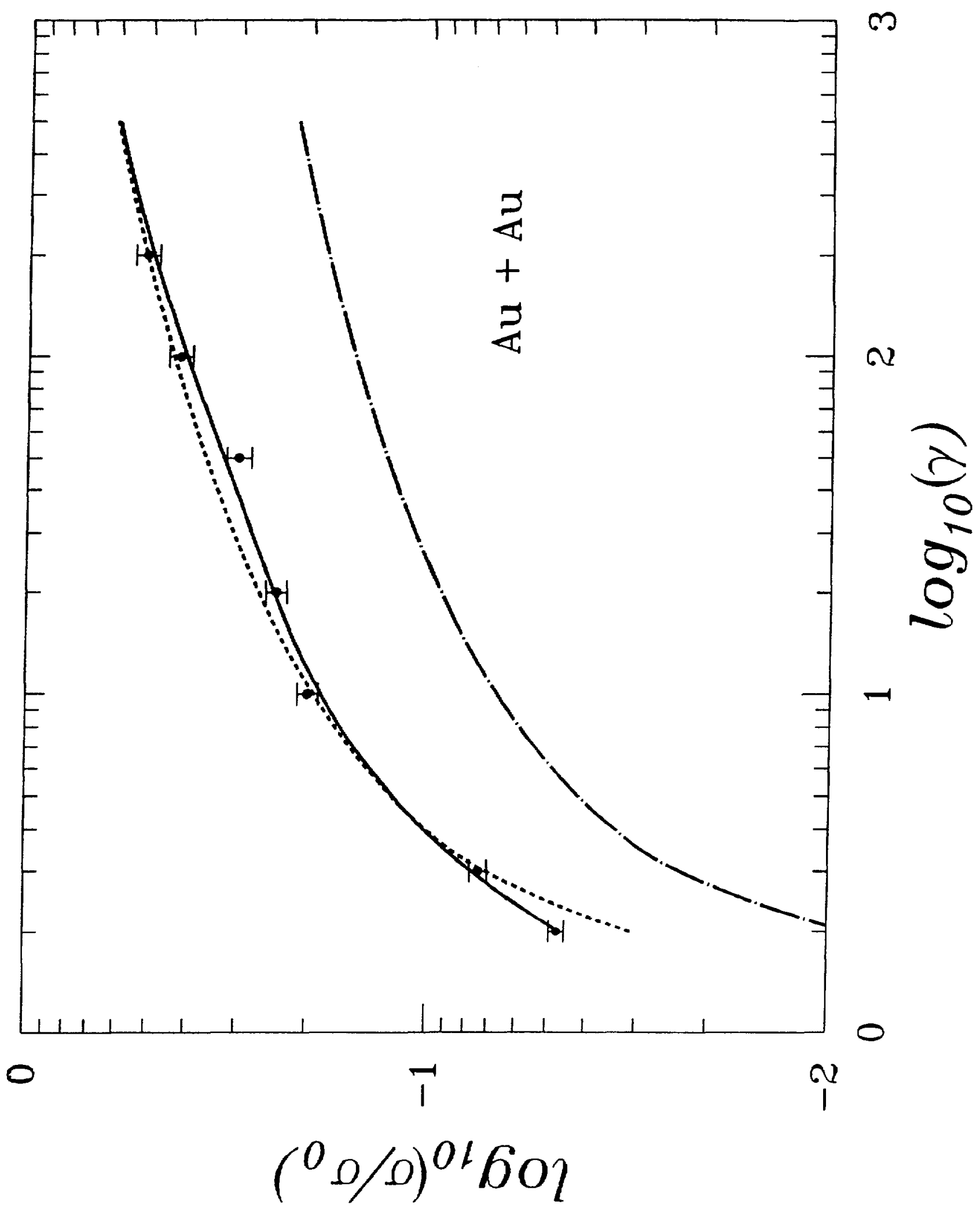

FIG: 


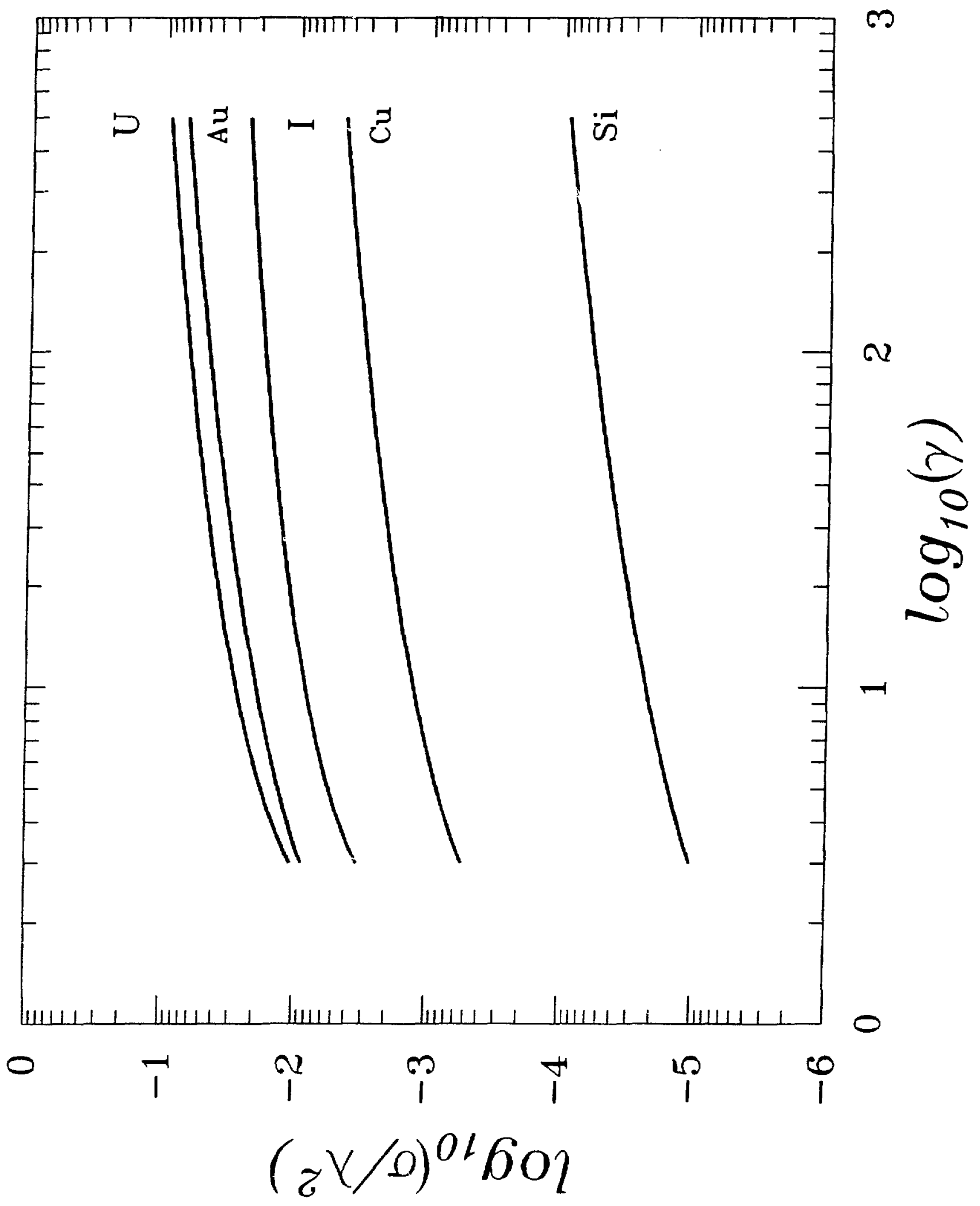

FIG. 3 


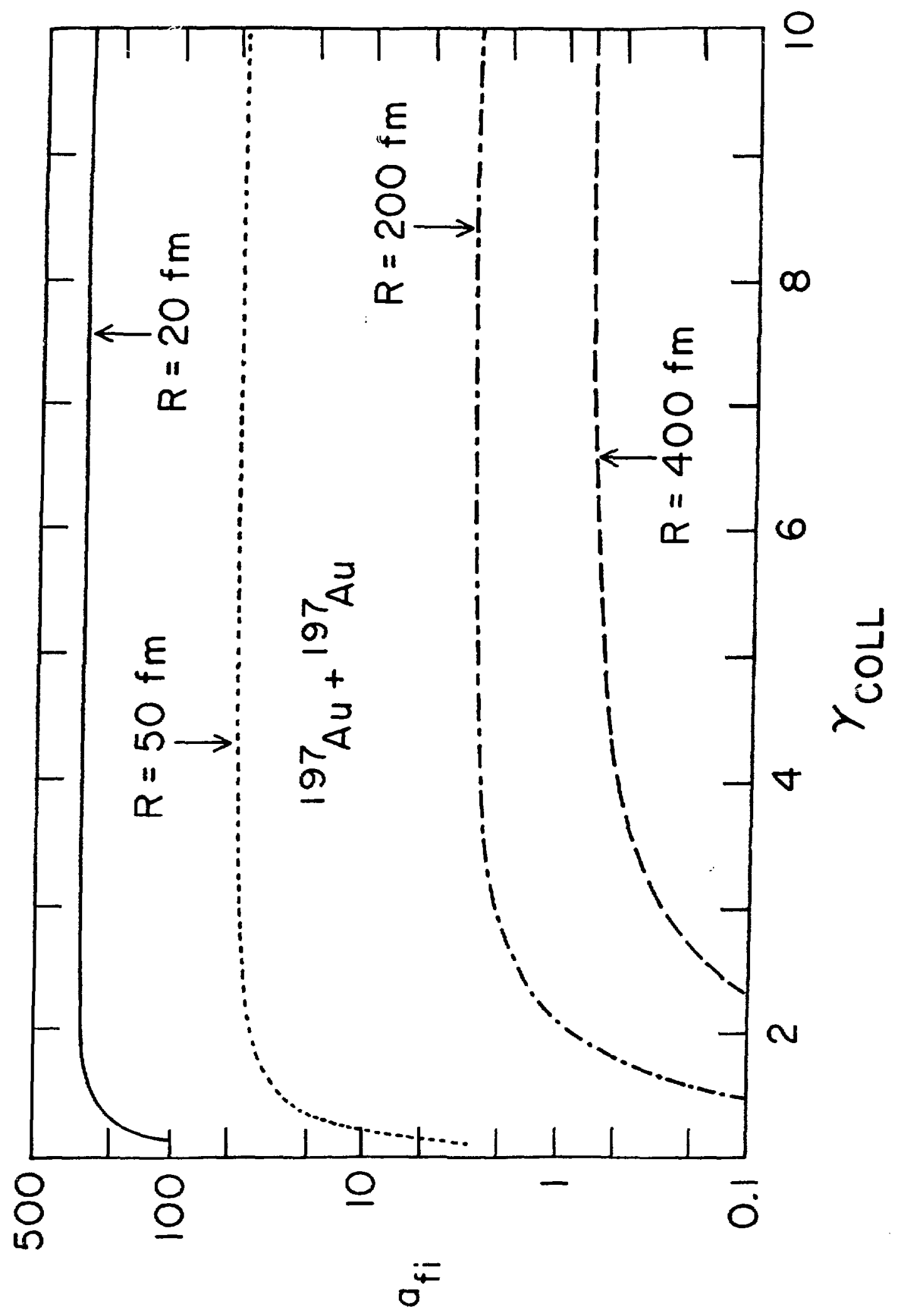




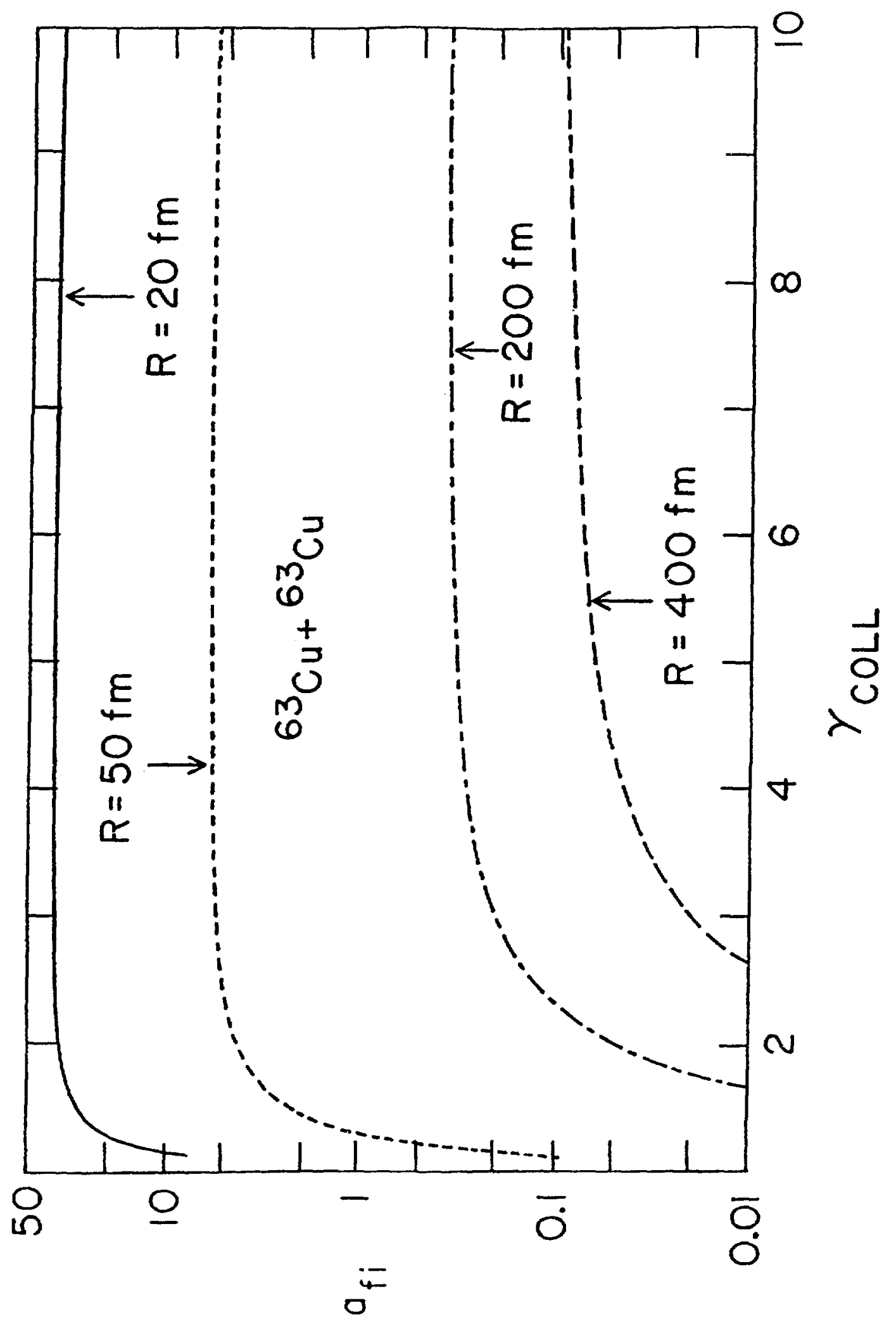

FIG. 5. 